
roseiras cultivadas em vaso. Horticultura Brasileira 26: 528-532.

\title{
Densidade de plantas e número de drenos influenciando a produtividade de roseiras cultivadas em vaso
}

\author{
Thales VA Viana'; Alexandre M Alves²; Valdemício F de Sousa ${ }^{3}$; Benito M de Azevedo ${ }^{4}$; Raquel Aparecida \\ Furlan $^{5}$ \\ ${ }^{1}$ UFC-Depto. Eng. Agrícola, C. Postal 12168, 60455-970 Fortaleza-CE; ${ }^{3}$ Embrapa Meio Norte, C. Postal 01, 64006-220 Teresina-PI; \\ ${ }^{4}$ UFC-DENA; ${ }^{2}$ Mestrando UFC; ${ }^{5}$ bolsista CNPq; thales@ufc.br; benito@ufc.br; raquel_furlan@yahoo.com.br
}

\begin{abstract}
RESUMO
O cultivo de flores no estado do Ceará vem se ampliando nos últimos anos, principalmente nas regiões serranas que proporcionam clima favorável ao desenvolvimento de diversas culturas. Entretanto, poucos são os trabalhos desenvolvidos nessa área, fazendo com que os produtores se utilizem do empirismo no desenvolvimento dos cultivos. Por conseguinte, esse trabalho teve como objetivo avaliar os efeitos do número de plantas $(2,3$ e 4) e da quantidade de drenos (1 e 8) por vaso no número de hastes por vaso da roseira, em ambiente protegido. O experimento foi conduzido na Empresa Reijers, no município de São Benedito-CE, sendo o delineamento experimental em blocos casualizados em arranjo fatorial $3 \times 2$, com quatro repetições. Avaliou-se o número de hastes com 35, 40, 50 e $60 \mathrm{~cm}$ e, o número total de hastes por vasos de $12,0 \mathrm{~L}$. As maiores quantidades de plantas por vaso proporcionaram um maior número de hastes por área, mas com predomínio de hastes de menor valor comercial. A utilização de um menor número de plantas por vaso resultou em um menor número de hastes por área, mas em maior número de hastes com maior valor comercial. A utilização de vasos com maior número de drenos reduziu o número total de hastes por vaso.
\end{abstract}

Palavras-chave: Rosa sp., floricultura, ambiente protegido.

\section{ABSTRACT}

Planting density and number of drains influencing the productivity of rose plants cultivated in pots

The cultivation of flowers in Ceará State has expanded during the last years, especially in highland areas with a favorable climate for several crops. However, there exist only limited research in this area, so that producers work empirically with those crops. Consequently, this research aimed to evaluate the effects of the number of plants (2, 3 and 4) and the amount of drains (1 and 8) per pot on the number of stems per pot, in a protected environment. The experiment was carried out in São Benedito, Ceará State, Brazil, following a $3 \times 2$ factorial randomized block design with four repetitions. The number of stems with $35,40,50,60 \mathrm{~cm}$ and, the total number of stems per pots of $12.0 \mathrm{~L}$ were evaluated. The highest number of plants per pot provided the greatest number of stems per pot, but with a predominance of lower commercial value. On the other hand, the least number of plants per pot resulted in few stems per area which were better evaluated commercially. The use of pots having more drains reduced the total number of stems per pot.

Keywords: Rosa $s p$., floriculture, protected environment.

(Recebido para publicação em 18 de outubro de 2007; aceito em 30 de outubro de 2008) (Received in October 18, 2007; accepted in October 30, 2008)

\begin{abstract}
A roseira (Rosa sp) pertencente à família Rosácea é cultivada desde os tempos remotos. Seu hábito de crescimento pode ser ereto, trepador ou reptante; as folhas são dispostas de forma alternada e as flores podem ocorrer de forma solitária ou em cacho, sendo o fruto do tipo aquênio (Folegatti et al., 2001; Barbosa, 2003).

A roseira sempre desempenhou papel de destaque entre as plantas ornamentais, sendo hoje uma das floríferas mais apreciadas no mundo (Folegatti et al, 2001; Seagri, 2002; Salagnac, 2003). O mercado mundial de flores e plantas ornamentais está em plena expansão e tem como principal exportador a Holanda, seguida pela Colômbia e pela Itália. O Brasil tem participação pouco expressiva no mercado mundial, mas esta participação vem se expandindo ao longo dos anos (Matsunaga, 1995; Rego et al., 2004).
\end{abstract}

No Ceará, a floricultura vem se destacando nos últimos anos, principalmente nas regiões serranas que proporcionam um clima favorável ao desenvolvimento de diversas culturas, dentre elas a roseira. O Estado começou a despertar para a produção comercial de rosas principalmente na região da serra da Ibiapaba, onde empresas de grande porte estão sendo instaladas, proporcionando um crescimento significativo desta atividade (Seagri, 2002).

$\mathrm{O}$ número de plantas por recipiente influencia a disponibilidade de água e nutrientes para as plantas. Normalmente, nos recipientes com menor densidade de plantas observa-se melhor arquitetura do sistema radicular (Parviainen, 1981), maior volume de raízes, melhor absorção de nutrientes e obtenção de plantas vigorosas com melhor qualidade. Entretanto, menores densidades de planta implicam em maiores custos para os produtores e, normalmente, em menores produtividades por área (Bezerra, 2003).

O maior número de plantas por vaso resulta em menor crescimento e vigor, por restringir o desenvolvimento do sistema radicular (Bezerra, 2003). Nesmith \& Duval (1998) relatam que a restrição das raízes afeta o crescimento, a fotossíntese, o teor de clorofila nas folhas, a absorção de água e nutrientes, a respiração, o florescimento e até a produção. Além disso, a maior quantidade de raízes em recipiente com pequena capacidade volumétrica, contribui para a redução do espaço poroso.

Segundo Costa et al. (2005), a drenagem consiste na retirada do excesso de água do solo a uma taxa que permita uma exploração econômica das cultu- 
ras e uma utilização por longo tempo da área. O teor de umidade disponível no solo, dentro de uma faixa ótima em que não há excesso ou déficit hídrico, constitui um dos parâmetros imprescindíveis para o desenvolvimento das culturas, facilitando o transporte de nutrientes através das raízes. Por outro lado, as condições de umidade excessiva na zona radicular são adversas para a maioria das culturas, devido à deficiência no teor de oxigênio no solo, comprometendo o transporte de nutrientes através do sistema radicular e tornando as plantas mais suscetíveis às doenças e à deficiência nutricional. Kanwar et al. (1988) ressaltam que a difusão do oxigênio através de poros cheios de ar é aproximadamente 10.000 vezes mais rápida que através de poros cheios de água; conseqüentemente, a taxa de difusão de oxigênio através da água é frequientemente o fator limitante da respiração das raízes.

Segundo Gervásio (2003), ao contrário dos cultivos em solo, o manejo da irrigação em recipientes pequenos como em vasos, apresenta algumas particularidades como maior frequiência de irrigação, em virtude do reduzido volume de substrato disponível para a planta. Essas particularidades implicam em maiores riscos de excesso hídrico, os quais devem ser prevenidos com um maior controle da irrigação. Além disso, a forma e o tamanho do recipiente influenciam a dinâmica da movimentação da água. De acordo com Fermino (2002), quanto menor a altura do recipiente, menos elevado será o fluxo de água em seu interior. Dessa forma, a reduzida altura de determinados recipientes costuma dificultar a drenagem, elevar a capacidade de retenção de água e, com isso, promover o encharcamento do substrato.

O excesso hídrico ocasiona a diminuição da pressão de oxigênio (hipoxia) ou a falta total ou parcial do mesmo (anoxia), dificultando a respiração das plantas e conseqüentemente, diminuindo a produção de energia necessária para a síntese e translocação dos compostos orgânicos e a absorção ativa dos mesmos. A falta de oxigênio nas raízes também provoca a redução na fotossíntese e prejudica a conversão da matéria orgânica, pelos microorganismos, em for- mas solúveis que a planta pode reutilizar. Ocorrendo, portanto, menor crescimento das plantas (Rego et al., 2004).

Basicamente, a falta de aeração constitui o principal agente de injúrias às plantas. Algumas evidências disponíveis indicam que efeitos deletérios são também produzidos pela atividade microbiológica em condições anaeróbicas. A extensão de danos causados às culturas, por tais condições adversas, varia com a espécie vegetal, a duração da inundação, o estádio de desenvolvimento, além da temperatura prevalecente à época da inundação (Costa et al., 1994).

Esse estudo teve como objetivo, avaliar os efeitos do número de plantas e da quantidade de drenos por vaso no número de hastes da roseira por vaso, em ambiente protegido.

\section{MATERIAL E MÉTODOS}

O experimento foi realizado de novembro de 2004 a fevereiro de 2005 na unidade de produção da agroempresa Reijers, localizada no sítio Lagoa, no município de São Benedito, CE (04 03'S, 40 53'W; $883 \mathrm{~m})$. O clima foi classificado, segundo a metodologia de Köppen, como Am, clima tropical chuvoso característico de áreas elevadas. $\mathrm{O}$ ambiente protegido utilizado no experimento apresentava $197 \mathrm{~m}$ de comprimento, $66 \mathrm{~m}$ de largura, totalizando 1,3 ha. A estrutura de sustentação era metálica em formato de arco, com pé-direito de 4,0 $\mathrm{m}$, abertura para saída de ar tipo lanternim e coberta por polietileno de baixa densidade (PEBD).

O experimento foi constituído por uma linha de vasos em fileira dupla, tendo cada uma 87 vasos, num total de 174. As plantas de roseiras utilizadas foram da cultivar akito; flor de coloração branca e botão pequeno, com ótima aceitação no mercado europeu. As mudas foram feitas em bandejas com fibras de mesocarpos de casca de coco queimado a partir de estacas com cerca de $5 \mathrm{~cm}$ de tamanho, que foram imersas por cinco segundos no hormônio AIB (ácido indolbutírico) a uma concentração de 2000 ppm, tendo sido transplantadas para os vasos aos 25 dias após o plantio.

Durante o desenvolvimento da cultura foram realizados tratos culturais como a desponta, que consistiu na retirada das primeiras folhas da muda para quebrar a dominância apical. Com o desenvolvimento da cultura realizou-se o agóbio, que consistiu no rebaixamento lateral da planta sem a danificação do caule, para a formação de uma massa foliar capaz de gerar hastes de qualidade. Realizaram-se também limpezas dos vasos, onde todo material vegetal depositado sobre o substrato foi retirado para evitar a decomposição e a incidência de doenças. Realizaram-se ainda desbrotas, onde se retiravam os brotos secundários evitando a deformação da haste, assim como a fixação de apenas um botão. Durante o ciclo foram realizadas aplicações preventivas de defensivos químicos para o controle de pragas e doenças.

As principais pragas e doenças identificadas que atuavam sobre a cultura na região foram o ácaro que é a praga mais comum sendo facilmente encontrada no interior das estufas, o tripes, pulgão, oídio, míldio e botrytis. Para o controle dessas pragas e doenças foram utilizados, principalmente, os defensivos malationa, oxicloreto de cobre, endosulfan, abamectin e dimethoate.

As plantas foram irrigadas por gotejamento, constituído por gotejadores com vazão de $1,0 \mathrm{~L} \mathrm{~h}^{-1}$, com um emissor por vaso. A irrigação foi realizada diariamente e era dividida em pulsos, onde cada pulso compreendia quinze minutos, totalizando uma aplicação diária de 4,0 mm da solução aquosa. Os vasos (volume de $12 \mathrm{~L}$ ) possuíam orifícios na parte inferior, com diâmetro de $1,0 \mathrm{~cm}$ para a drenagem do excesso de água, que era reaproveitada.

A adubação foi realizada diariamente via fertirrigação. As quantidades aplicadas foram definidas por meio de análises químicas e de fertilidade do substrato e das folhas.

O delineamento experimental foi em blocos casualizados, em arranjo fatorial $3 \times 2$, onde cada tratamento apresentava quatro parcelas constituídas de seis vasos, totalizando vinte e quatro. Foram avaliados três quantidade de plantas por vaso (2, 3 e 4) sob duas quantidades de dreno (1 e 8). As bordaduras entre as parcelas constituíram-se de quatro vasos, deixando-se ainda cinco vasos como bordaduras no final e no início da linha. 
Tabela 1. Números de hastes de $35,40,50$ e $60 \mathrm{~cm}$ e total/vaso/ciclo sob as densidades de 2 , 3 e 4 plantas/vaso (number of stems of 35, 40, 50, and $60 \mathrm{~cm}$ and total/pot/cycle under densities of 2, 3, and 4 plants/pot). São Benedito, UFC, 2005.

\begin{tabular}{llllll}
\hline Plantas por & \multicolumn{5}{c}{ Hastes por vaso por ciclo } \\
\cline { 2 - 6 } vaso & $\mathbf{3 5} \mathbf{~ c m}$ & $\mathbf{4 0} \mathbf{~ c m}$ & $\mathbf{5 0} \mathbf{~ c m}$ & $\mathbf{6 0} \mathbf{~ c m}$ & Total \\
\hline 2 & $0,13 \mathrm{~B}$ & $2,04 \mathrm{~B}$ & $2,63 \mathrm{~A}$ & $0,45 \mathrm{~A}$ & $5,25 \mathrm{~B}$ \\
3 & $0,50 \mathrm{~A}$ & $3,46 \mathrm{~A}$ & $2,04 \mathrm{~B}$ & $0,42 \mathrm{~A}$ & $6,42 \mathrm{~A}$ \\
4 & $0,58 \mathrm{~A}$ & $3,04 \mathrm{~A}$ & $2,21 \mathrm{~B}$ & $0,21 \mathrm{~A}$ & $6,04 \mathrm{~A}$ \\
$\mathrm{~F}$ & $0,00641^{* *}$ & $0,00001^{* *}$ & $0,00412^{* *}$ & n.s & $0,0004^{* *}$ \\
\hline C.V. $(\%)$ & 17,4 & 9,3 & 14,7 & 19,8 & 16,5 \\
\hline DMS & 0,32 & 0,43 & 0,37 & - & 0,68 \\
\hline
\end{tabular}

Médias seguidas da mesma letra na coluna não diferem entre si pelo teste de Tukey $5 \%$ (means followed by the same letter in the column did not differ from each other, Tukey's test, $\mathrm{p} \leq 0,05)$.

Tabela 2. Número de hastes de 35, 40, 50 e $60 \mathrm{~cm}$ e total/vaso/ciclo sob 1 e 8 drenos por vaso (number of stems of 35, 40,50, and $60 \mathrm{~cm}$ and total/pot/cycle under 1 and 8 drains per pot). São Benedito, UFC, 2005.

\begin{tabular}{lclccc}
\hline Plantas por & \multicolumn{5}{c}{ Hastes por vaso por ciclo } \\
\cline { 2 - 6 } vaso & $\mathbf{3 5} \mathbf{~ c m}$ & $\mathbf{4 0} \mathbf{~ c m}$ & $\mathbf{5 0} \mathbf{~ c m}$ & $\mathbf{6 0} \mathbf{~ c m}$ & Total \\
\hline 1 & $0,39 \mathrm{~A}$ & $3,33 \mathrm{~A}$ & $2,36 \mathrm{~A}$ & $0,28 \mathrm{~A}$ & $6,36 \mathrm{~A}$ \\
8 & $0,41 \mathrm{~A}$ & $2,36 \mathrm{~B}$ & $2,22 \mathrm{~A}$ & $0,45 \mathrm{~A}$ & $5,44 \mathrm{~B}$ \\
\hline $\mathrm{F}$ & n.s & $0,00113^{* *}$ & n.s & n.s & $0,0005^{* *}$ \\
\hline C.V. (\%) & 16,7 & 9,8 & 12,3 & 18,7 & 15,9 \\
\hline DMS & - & 0,56 & - & - & 0,34 \\
\hline
\end{tabular}

Médias seguidas da mesma letra na coluna não diferem entre si pelo teste de Tukey $5 \%$ (means followed by the same letter in the column did not differ from each other, Tukey's test, $\mathrm{p} \leq 0,05)$.

Os tratamentos foram nomeados em conformidade com a quantidade de plantas e o número de drenos por vaso, ou seja, o tratamento 3P1D correspondia a três plantas e um dreno por vaso, o 3P8D correspondia a três plantas e oito drenos por vaso e, assim, sucessivamente.

A colheita das rosas foi realizada dos 52 aos 59 dias após o transplantio (DAT), em conformidade com o padrão para exportação das hastes (quando as 4 primeiras pétalas desprendiam-se do botão floral). Foram avaliados os números de hastes de $35,40,50$ e $60 \mathrm{~cm}$ e o número total de hastes por vaso.

Os dados foram submetidos à análise de variância (ANOVA) e quando significativo pelo teste $\mathrm{F}$ usou-se o teste de Tukey a $5 \%$ de probabilidade. As análises foram realizadas por meio do programa estatístico SAEG.

\section{RESULTADOS E DISCUSSÃO}

Na Tabela 1 são apresentados os números de hastes de $35,40,50$ e 60 tas por vaso aumenta o desenvolvimento das mesmas (Parviainen, 1981; Nesmith \& Duval, 1998), muitos ressaltam uma redução na produção por área (Nesmith \& Duval, 1998; Bezerra, 2003), nesse caso no número de hastes por vaso. Por conseguinte, nos vasos com mais plantas, e consequientemente menos substrato por planta, a maior competição por nutrientes resultou em hastes menores mas, como o número de plantas era maior, a produtividade total também foi maior.

A produção total foi menor nos vasos com menos plantas, mas a produção de hastes de maior valor de mercado $(50$ e $60 \mathrm{~cm}$ ) foi maior (Tabela 1). Provavelmente, a maior quantidade de substrato disponível por planta permitiu o deslocamento de maior quantidade de nutrientes, possibilitando o desenvolvimento de hastes de maior comprimento. Relato semelhante fizeram Luz et al. (2000).

Em síntese, com relação às densidades de plantas/vaso, pode-se afirmar que maiores densidades implicaram em maior número de hastes por área, mas com predomínio de hastes de menor valor comercial. Já a utilização de menos plantas por vaso resultou em menor número de hastes por área, mas em maior quantidade de hastes de maior valor comercial.

Na Tabela 2 são apresentados os valores observados para o número de hastes de 35, 40, 50 e $60 \mathrm{~cm}$ e total/vaso/ ciclo sob 1 e 8 drenos por vaso. Houve diferença significativa entre os tratamentos somente para o número de hastes de $40 \mathrm{~cm}$ por vaso e o número total de hastes por vaso.

Com relação ao número de hastes de $40 \mathrm{~cm}$ por vaso, o melhor resultado foi encontrado no tratamento com um dreno. Resultado semelhante foi obtido também com a variável número total de hastes por vaso (Tabela 2). Esses resultados demonstram que provavelmente a utilização de um dreno mantém disponível por maior tempo uma maior quantidade de água e nutrientes para as plantas, em relação à utilização de oito drenos, possibilitando a melhor nutrição 
das plantas. Isso provavelmente acontece porque o substrato não apresenta uma estruturação significativa visto que o mesmo é substituído no início dos cultivos. Essa explicação corrobora com os comentários feitos por Fermino (2002) e Gervásio (2003).

Os resultados da análise de variância com relação à interação número de plantas versus número de drenos por vaso estão apresentados na Tabela 3. Todas as variáveis apresentaram diferenças significativas.

A maior produção de hastes de 35 cm por vaso ocorreu com os tratamentos 4P8D (quatro plantas e oito drenos por vaso) e 3P1D. Já a produção de hastes de $40 \mathrm{~cm}$ foi mais evidenciada somente em 3P1D. Para as hastes de $50 \mathrm{~cm}$, o melhor desempenho ocorreu com o tratamento $2 \mathrm{P} 1 \mathrm{D}$, que não diferiu estatisticamente dos tratamentos 2P8D, 4P1D e 3P8D. Com relação às hastes de $60 \mathrm{~cm}$ de comprimento, as de maior valor de mercado, a menor produção foi observada com o tratamento 4P8D. Em complemento, o número total de haste por vaso apresentou o maior valor no tratamento 3P1D (Tabela 3).

De modo geral, o tratamento 3P1D produziu o maior número de hastes por vaso, mas com supremacia nas hastes de menor tamanho. Caso ao produtor seja mais interessante a quantidade e não a qualidade, o mesmo deve optar por este tratamento. Caso ele deseje hastes maiores $(50$ e $60 \mathrm{~cm}$ ), o produtor pode optar por 2 plantas/vaso. Resultado semelhante quanto à densidade de plantas foi obtido por Folegatti et al. (2001).

Em síntese, a utilização de 3 plantas e 1 dreno por vaso permite a maior produção total por hastes. Nessa condição, há maior competição por nutrientes, em comparação com a condição 2 plantas/ vaso, implicando em hastes menores, mas em maior número, devido ao maior número de plantas por vaso. Redução no crescimento das plantas em função da redução da disponibilidade de nutrientes também foi relatada por Carneiro (1983), Backes \& Kãmpf (1991), Mascarenhas (1993), Nesmith \& Duval (1998) e Bezerra (2003). Por outro lado, a utilização de um só dreno em vasos deve aumentar

Tabela 3. Número de hastes de 35, 40, 50 e $60 \mathrm{~cm}$ e total/vaso/ciclo nos diferentes tratamentos (number of stems of $35,40,50$, and $60 \mathrm{~cm}$ and total/pot/cycle in different treatments). São Benedito, UFC, 2005.

\begin{tabular}{llllll}
\hline Plantas por & \multicolumn{5}{c}{ Hastes por vaso por ciclo } \\
\cline { 2 - 6 } vaso & $\mathbf{3 5} \mathbf{~ c m}$ & $\mathbf{4 0} \mathbf{~ c m}$ & $\mathbf{5 0} \mathbf{~ c m}$ & $\mathbf{6 0} \mathbf{~ c m}$ & Total \\
\hline 4P1D & $0,42 \mathrm{AB}$ & $3,33 \mathrm{~B}$ & $2,42 \mathrm{AB}$ & $0,33 \mathrm{AB}$ & $6,50 \mathrm{~B}$ \\
4P8D & $0,75 \mathrm{~A}$ & $2,75 \mathrm{BC}$ & $2,00 \mathrm{~B}$ & $0,08 \mathrm{~B}$ & $5,58 \mathrm{C}$ \\
3P1D & $0,75 \mathrm{~A}$ & $4,33 \mathrm{~A}$ & $1,92 \mathrm{~B}$ & $0,25 \mathrm{AB}$ & $7,25 \mathrm{~A}$ \\
3P8D & $0,25 \mathrm{~B}$ & $2,58 \mathrm{C}$ & $2,17 \mathrm{AB}$ & $0,58 \mathrm{AB}$ & $5,58 \mathrm{C}$ \\
2P1D & $0,00 \mathrm{~B}$ & $2,33 \mathrm{CD}$ & $2,75 \mathrm{~A}$ & $0,25 \mathrm{AB}$ & $5,33 \mathrm{C}$ \\
2P8D & $0,25 \mathrm{~B}$ & $1,75 \mathrm{D}$ & $2,50 \mathrm{AB}$ & $0,67 \mathrm{~A}$ & $5,17 \mathrm{C}$ \\
\hline F & $0,00004^{* *}$ & $0,0000^{* *}$ & $0,01112^{*}$ & $0,01871^{*}$ & $0,0000^{* *}$ \\
\hline C.V. (\%) & 18,5 & 9,7 & 14,2 & 20,2 & 17,4 \\
\hline DMS & 0,42 & 0,65 & 0,72 & 0,53 & 0,91 \\
\hline
\end{tabular}

Médias seguidas da mesma letra na coluna não diferem entre si pelo teste de Tukey $5 \%$ (means followed by the same letter in the column did not differ from each other, Tukey's test, $\mathrm{p} £ 0,05)$.

o período em que a solução fertirrigante fica disponível às raízes das plantas aumentando a absorção dos nutrientes e, conseqüentemente, dos diferentes processos fisiológicos dependentes dos macro e micronutrientes contidos na solução fertirrigante. Redução da intensidade de drenagem em função de um menor número de drenos, também foi relatada por Costa et al. (2005).

Pode-se concluir que as maiores densidades de plantas por vaso proporcionaram maior número de hastes por área, mas com predomínio de hastes de menor valor comercial. A utilização de menores densidades de plantas por vaso resultou em menor número de hastes por área, mas em maior número de hastes com maior valor comercial. A utilização de vasos com maior número de drenos reduziu o número total de hastes por vaso.

\section{REFERÊNCIAS}

BACKES MA; KÄMPF AN. 1991. Substrato à base de composto de lixo urbano para a produção de plantas ornamentais. Pesquisa Agropecuária Brasileira 26: 753-758.

BARbOSA JG. 2003. Produção Comercial de Rosas. Viçosa: Aprenda Fácil. 200p.

BEZERRA FC. 2003. Produção de mudas de hortaliças em ambiente protegido. Fortaleza: Embrapa Agroindústria Tropical. 22 p. (Documentos, 72).

CARNEIRO JGA. 1983. Variações na metodologia de produção de mudas florestais afetam os parâmetros morfo-fisiológicos que indicam a sua qualidade. Curitiba: FUPEF (Série Técnica) 12:1-40.
COSTA RNT; COLARES DS; SAUNDERS LCU; SOUZA F. 2005. Análise das eficiências de aplicação e de uso da água em cultivo de arroz no Perímetro Irrigado Morada Nova, Ceará. Irriga 10: 372-382.

COSTA RNT; SAUNDERS LCU; FERREYRA HFF. 1994. Efeito da associação de tratamentos mecânicos, químico e vegetativo em um solo sódico sob condições de drenagem subsuperficial no vale do Curu-Ce. Engenharia Rural 5:1-19.

FERMINO MH. 2002. O uso da análise física na avaliação da qualidade de componentes e substratos. In: ENCONTRO NACIONAL DE SUBSTRATO PARA PLANTAS, 2. Anais... Campinas: IAC, p. 29-37.

FOLEGATTI MV; CASARINI E; BLANCO FF. 2001. Lâminas de irrigação e a qualidade de hastes e de botões florais de rosas cultivadas em ambiente protegido. Scientia Agricola 58: 465-468.

GERVÁSIO ES. 2003. Efeito de lâminas de irrigação e doses de condicionador, associadas a diferentes tamanhos de tubetes, na produção de mudas de cafeeiro. Piracicaba: USP-ESALQ. 105p (Tese doutorado).

KANWAR RS; BAKER J1; MUKHTAR S. 1988. Excessive soil water effects at various stages of development on the growth and yield of corn. St. Joseph: Transaction of the ASAE 31: 133-141.

LUZ JMQ; PAULA EC; GUIMARÃES TG. 2000. Produção de mudas de alface, tomateiro e couve-flor em diferentes substratos comerciais. Horticultura Brasileira 18: 579-580.

MASCARENHAS MHT. 1993. Cebola. Informe Agropecuário 14: 69-73.

MATSUNAGA M. 1995. Potencial da floricultura brasileira. Agroanalysis 15: 56.

NESMITH DS; DUVAL JR. 1998. The effect of container size. Hort Technology 8: 495-498.

PARVIAINEN JV. 1981. Qualidade e avaliação de qualidade de mudas florestais. In: SEMINÁRIO DE SEMENTES E VIVEIROS FLORESTAIS, 1. Anais... Curitiba: FUPEF, p. 59-90. 
REGO JL; VIANA TVA; AZEVEDO BM; BASTOS FGC; GONDIM RS. 2004. Efeitos de níveis de irrigação sobre a cultura do crisântemo. Revista Ciência Agronômica 35: 302-308.
SALAGNAC C. Exportação: floricultura. 2003. Disponível em: <http://www.panrural.com.br/ ver_noticia.asp?news_id=76 > Acessado em: 17 de outubro de 2005.
SEAGRI - Secretaria de Agricultura Irrigada. 2002. Agronegócio da floricultura no Estado do Ceará. . Disponível em <http://www5.prossiga.br/ arranjos/vortais/floricultura_ce_oquee001.html, http://www5 5rossiga.br/arranjos/vortais/ floricultura_ce_oquee002.html > Acessado em 17 de outubro de 2005 . 